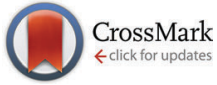

Cite this: J. Mater. Chem. C, 2016 4, 11305

Received 20th October 2016, Accepted 3rd November 2016

DOI: $10.1039 / \mathrm{c} 6 \mathrm{tc0} 4553 \mathrm{~g}$

www.rsc.org/MaterialsC

\section{Stability, electronic structures and thermoelectric properties of binary $\mathrm{Zn}-\mathrm{Sb}$ materials}

\author{
Xin $\mathrm{He}^{a}{ }^{a}$ Yuhao Fu, ${ }^{a}$ David J. Singh ${ }^{\star a b}$ and Lijun Zhang ${ }^{{ }^{a}}$
}

\begin{abstract}
We report first principles studies of the binary $\mathrm{Zn}-\mathrm{Sb}$ phases in relation to thermoelectric properties and chemical stability. We identify the unknown structure of the $\mathrm{Zn}_{3} \mathrm{Sb}_{2}$ phase using particle swarm optimization, finding a tetragonal structure different from the hexagonal $\mathrm{Mg}_{3} \mathrm{Sb}_{2}$ and the hexagonal or $\mathrm{cubic} \mathrm{Ca}_{3} \mathrm{Sb}_{2}$ phases. All the phases are found to be semiconducting with bandgaps in the range of $0.06-0.77 \mathrm{eV}$. This semiconducting behavior is understood in Zintl terms as a balance between the $\mathrm{Zn}: \mathrm{Sb}$ and $\mathrm{Sb}^{3-}: \frac{1}{2}\left(\mathrm{Sb}_{2}\right)^{4-}$ ratios in the stable crystal structures. With the exception of $\mathrm{Zn}_{3} \mathrm{Sb}_{2}$, which has a small gap, all the compounds have electronic properties favorable for thermoelectric performance.
\end{abstract}

\section{Introduction}

Thermoelectric (TE) devices can directly convert between electrical and thermal energies for cooling or heating or to recover waste heat. These materials have traditionally had an important role in energy conversion, especially portable refrigeration devices, and power generation especially for space crafts. ${ }^{1-3}$ They are increasingly seen as having the potential to make important contributions to energy technology more generally ${ }^{3-5}$ and have additionally attracted fundamental interest due to the connection with topological insulators and the possibility of new types of thermoelectrics. ${ }^{5-9}$ Furthermore there has been interest in transient thermoelectric effects. ${ }^{10-15} \mathrm{~A}$ key challenge for enabling applications is to improve the TE properties of the active materials.

Efficiency plays a central role in energy technology. In the case of TE, this is limited by a figure of merit $Z T=\sigma S^{2} T / k$, where $\sigma$ is the electrical conductivity, $S$ is the thermopower, $k$ is the thermal conductivity, and $T$ is the absolute temperature. A good TE material should have excellent electrical transport properties, measured by the TE power factor $\left(\mathrm{PF}=S^{2} \sigma\right)$ and also a low thermal conductivity $k$.

Caillat et al. found a high $Z T$ of 1.3 at $970 \mathrm{~K}$ in p-type $\beta-\mathrm{Zn}_{4} \mathrm{Sb}_{3}$ in $1997 .{ }^{16}$ The high performance combined with the relatively inexpensive, environmentally acceptable composition stimulated considerable interest. Four compounds in the narrow range of $50-60$ atomic\% of $\mathrm{Zn}$ were reported in the binary $\mathrm{Zn}-\mathrm{Sb}$ phase diagram: $\mathrm{ZnSb}$ is a stoichiometric

\footnotetext{
${ }^{a}$ College of Materials Science and Engineering and Key Laboratory of Automobile Materials of MOE, Jilin University, Changchun 130012, China.

E-mail: lijun_zhang@jlu.edu.cn

${ }^{b}$ Department of Physics and Astronomy, University of Missouri, Columbia,

MO 65211-7010, USA. E-mail: singhdj@missouri.edu
}

compound with a well characterised crystal structure (CdSb-type) ${ }^{17}$ and stable up to $822 \mathrm{~K}$. $\mathrm{Zn}_{4} \mathrm{Sb}_{3}$ displays temperature dependent polymorphism, with a $\beta-\alpha$ transition at approximately $250 \mathrm{~K} \cdot{ }^{18,19}$ Two phases of $\mathrm{Zn}_{8} \mathrm{Sb}_{7}$ have been reported, $\alpha$ and $\beta{ }^{20,21} \mathrm{Zn}_{3} \mathrm{Sb}_{2}$ is metastable and has been reported without any crystal structure solution. ${ }^{22}$ Interestingly, the experimental values and temperature dependences of $S$ and $\sigma$ are very similar for $\mathrm{ZnSb}, \beta-\mathrm{Zn}_{4} \mathrm{Sb}_{3}$ and $\beta-\mathrm{Zn}_{8} \mathrm{Sb}_{7}$. What makes the $\beta$-phase special is the exceptionally low thermal conductivity, which is as low as that expected for glass-like materials. The presence of the interstitial $\mathrm{Zn}$ atoms in the $\beta$-phase crystal structures is the key to the low lattice thermal conductivity. ${ }^{20,23}$ There have been several studies of electrical and transport properties for $\beta-\mathrm{Zn}_{4} \mathrm{Sb}_{3}$, $\alpha-\mathrm{Zn}_{8} \mathrm{Sb}_{7}$ and $\mathrm{ZnSb},{ }^{24-32}$ including first principles studies of the electronic structures of these binaries ${ }^{26,30-32}$ and the related Zintl phases $\mathrm{SrZn}_{2} \mathrm{Sb}_{2}$ and $\mathrm{YbZn}_{2} \mathrm{Sb}_{2}$. However, the other phases have not been studied in similar detail. Here we report detailed firstprinciples calculations to establish the electronic structures of $\mathrm{Zn}$-Sb binary system compounds, elucidate the structures and bonding, and to assess their thermoelectric potential.

\section{Computational methodology}

Full structure optimizations were performed using the projector augmented wave $(\mathrm{PAW})^{33}$ method with generalized approximation (GGA) of Perdew-Burke-Ernzerhof (PBE) as implemented in the VASP code. ${ }^{34}$ Following a series of test calculations a plane-wave cutoff of $360 \mathrm{eV}$ and $k$-point meshes of spacing $2 \pi \times 0.03 \AA^{-1}$ or better were adopted. In all of the calculations the convergence limit was set to $1 \times 10^{-4} \mathrm{eV} \AA^{-1}$ for the force and $1 \times 10^{-5} \mathrm{eV}^{-1}$ for the energy. Standard GGA methods underestimate the band gaps due to the presence of artificial self-interaction and the absence of the derivative discontinuity 
in the exchange-correlation potential. ${ }^{35,36}$ We used the modified Becke Johnson (MBJ) ${ }^{37}$ potential to obtain more accurate bandgaps and optical properties. This potential generally improves the accuracy of the gap values relative to experiments. For example, the band gaps calculated using MBJ potential for $\mathrm{ZnSb}$ and $\beta-\mathrm{Zn}_{4} \mathrm{Sb}_{3}$ (0.60 and $0.77 \mathrm{eV}$, respectively) are much closer to experimental values (0.61 and $\left.0.8 \mathrm{eV}^{38,39}\right)$ than PBE values $(0.15$ and $0.4 \mathrm{eV})$.

This is important for predicting the thermoelectric properties, as the thermopower in particular can be strongly reduced by spurious bipolar effects from underestimated band gaps. However, the MBJ potential does not have a corresponding energy functional and therefore cannot be used to predict the structures. Therefore we use the PBE functional, which is designed to produce reliable total energies and structures, but not band gaps, to obtain crystal structures, and then do calculations with the $\mathrm{MBJ}$ potential to obtain band structures and transport properties. We did not include the spin-orbit coupling (SOC) effect in transport calculations since it shows a negligible effect on the electronic structures of the studied system. The values of the gaps are given in Table 1 along with calculated transport coefficients at $400 \mathrm{~K}$. The available experimental and calculated data are listed for comparison. The transport coefficients are discussed for each compound in the sections below.

In order to demonstrate the validity of using the MBJ potential, we calculate the optical spectra shown in Fig. 1. The onset of absorption is at $0.85 \mathrm{eV}$. The experimental onset of strong absorption transition is at approximately $1.05 \mathrm{eV}$. We find noticeable anisotropy in the optical absorption, very close to the onset. It shows significantly stronger absorption for polarization along $\mathrm{c}$, in accord with the experiment. ${ }^{38} \mathrm{We}$ predict that this anisotropy switches at higher energy so that

Table 1 Calculated indirect and direct bandgaps $E_{\mathrm{g}}$ (in eV), as well as thermopowers $S$ (in $\mu \mathrm{V} \mathrm{K}^{-1}$ ) and power factor divided by scattering time $\sigma S^{2} / \tau$ (in $10^{10} \mathrm{~W} \mathrm{~K}^{-2} \mathrm{~ms}$ ) at a temperature of $400 \mathrm{~K}$ and a carrier concentration of $10^{20} \mathrm{~cm}^{-3}$. The available experimental (Exp.) and calculated (Cal.) results are listed for comparison; for $\mathrm{S}$, the data for comparison are: $\mathrm{ZnSb}$ at a carrier concentration of $0.75 \times 10^{20} \mathrm{~cm}^{-3}$ at a temperature of $400 \mathrm{~K}$ from ref. 29 , and $1 \times 10^{20} \mathrm{~cm}^{-3}$ at $600 \mathrm{~K}$ from ref. $31 ; \beta-\mathrm{Zn}_{4} \mathrm{Sb}_{3}$ at $4.9 \times 10^{20} \mathrm{~cm}^{-3}$ at $400 \mathrm{~K}$ from ref. 29 , and $0.75 \times 10^{20} \mathrm{~cm}^{-3}$ at $400 \mathrm{~K}$ from ref. $26 ; \alpha-\mathrm{Zn}_{8} \mathrm{Sb}_{7}$ at $4.7 \times 10^{20} \mathrm{~cm}^{-3}$ at $400 \mathrm{~K}$ from ref. 29

\begin{tabular}{lllll}
\hline Compound & $\begin{array}{l}E_{\mathrm{g}} \\
\text { (indirect) }\end{array}$ & $\begin{array}{l}E_{\mathrm{g}} \\
(\text { direct })\end{array}$ & $\begin{array}{l}S(400 \mathrm{~K}, \\
\left.10^{20} \mathrm{~cm}^{-3}\right)\end{array}$ & $\begin{array}{l}\sigma S^{2} / \tau(400 \mathrm{~K}, \\
\left.10^{20} \mathrm{~cm}^{-3}\right)\end{array}$ \\
\hline $\mathrm{ZnSb}$ & $\begin{array}{l}0.60 \\
0.61(\text { Exp.) })^{38}\end{array}$ & 0.88 & $\begin{array}{l}98 \\
90(\text { Cal. })^{29}\end{array}$ & 7.3 \\
& $0.56(\text { Cal. })^{31}$ & & $135(\text { Cal. })^{31}$ & \\
$\alpha-\mathrm{Zn}_{4} \mathrm{Sb}_{3}$ & 0.68 & 0.77 & 205 & 14.5 \\
& 0.77 & & 161 & \\
$\beta-\mathrm{Zn}_{4} \mathrm{Sb}_{3}$ & $0.8(\text { Exp. })^{39}$ & 0.81 & $110(\text { Cal. })^{29}$ & 15.9 \\
& $0.3(\text { Cal. })^{30}$ & & $180(\text { Cal. })^{26}$ & \\
$\alpha-\mathrm{Zn}_{8} \mathrm{Sb}_{7}$ & 0.71 & 0.76 & 164 & 7.5 \\
$\beta-\mathrm{Zn}_{8} \mathrm{Sb}_{7}$ & 0.61 & 0.61 & 129 & 8.1 \\
$\mathrm{Zn}_{3} \mathrm{Sb}_{2}$ & 0.06 & 0.1 & 109 & 6.9
\end{tabular}

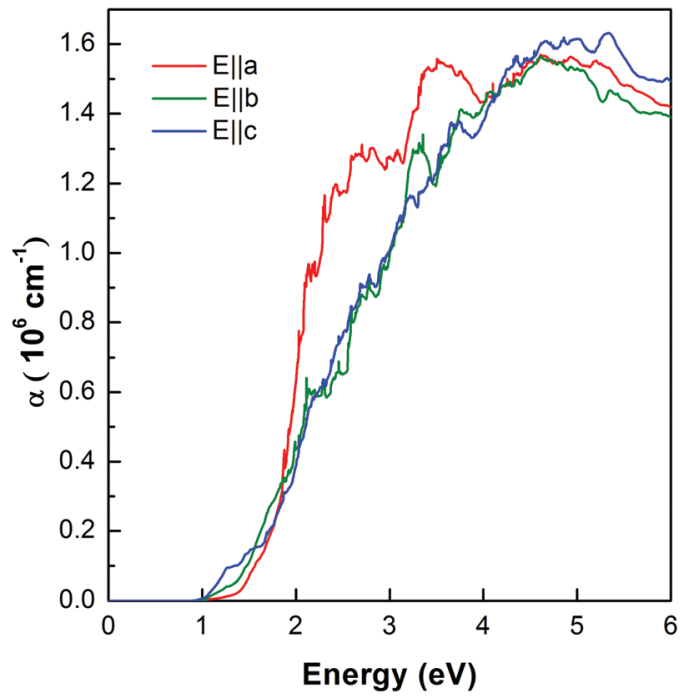

Fig. 1 Optical spectra of ZnSb calculated by using the MBJ functional.

the strongest absorption is for polarization along a in the range $2-4 \mathrm{eV}$.

The transport calculations were done using the BoltzTraP program. ${ }^{40}$ We used approximately $5000 k$-points in the irreducible Brillouin Zone, with the constant relaxation time approximation. The necessary crystal structure and eigenenergies were obtained from VASP results by using MBJ potential. This approach uses a rigid band approximation. This is generally valid for semiconductors that have good conductivity when doped, as is the case for the $\mathrm{Zn}$-Sb phases (note that strong band distortion upon doping is indicative of strong electronic scattering by dopants). It is also consistent with spectroscopic measurements for some of these phases in relation to prior density functional calculations. ${ }^{30-32}$ Going beyond the rigid band approximation would require knowledge of the specific doping elements and defects that occur in actual samples.

Previous studies on the phase diagram of the zinc-antimony system have reported compound $\mathrm{Zn}_{3} \mathrm{Sb}_{2} \cdot{ }^{41,42}$ But it is metastable and crystals amorphize over time, which impedes its structure and property characterization. We performed a crystal structure prediction for $\mathrm{Zn}_{3} \mathrm{Sb}_{2}$ via global minimization of free energy surfaces as implemented in the CALYPSO code, which searches for the stable structures of the given compounds. Using particle swarm optimization (PSO) the most stable structures based on the chemical composition can be found. Importantly, this approach is unbiased by already known structures. ${ }^{43,44}$ In this sense it differs from approaches such as those used in most materials genome projects, for example, the materials project, ${ }^{45}$ where known structures and structure types are generally relied upon. The advantage of the PSO approach is that it can find structures that were previously unknown, while on the other hand it requires calculations for many structures during the global optimization. Structure searching was performed at zero pressure with simulation cells consisting of 1-4 formula units (f.u.). This global optimization 
involved density functional relaxations of approximately 1500 distinct structures to find the ground state. The most stable predicted structure was then further optimized using VASP as described above. This calculation adds the structure and energy of $\mathrm{Zn}_{3} \mathrm{Sb}_{2}$ to the phase diagram.

\section{Structure and phase diagram}

We consider all $\mathrm{Zn}-\mathrm{Sb}$ binary materials for which structures have been reported and $\mathrm{Zn}_{3} \mathrm{Sb}_{2}$ with a predicted structure. Orthorhombic $\mathrm{ZnSb}$ (space group Pbac) was reported in 1948. ${ }^{17}$ It may be regarded as an arrangement of $\mathrm{Zn}$ centered, vertex and corner sharing $\mathrm{Sb}_{4}$ tetrahedra (quasitetrahedral close-packing), as shown in Fig. 2a. The formal valence state of $\mathrm{Sb}$ is -2 , and it has the appearance of a classical Zintl phase with $\mathrm{Sb}$ present as $\left(\mathrm{Sb}_{2}\right)^{4-}$ dimers. The $\mathrm{ZnSb}_{4}$ tetrahedra are linked by $\mathrm{Sb}-\mathrm{Sb}$ bonds. We note that such $\mathrm{Sb}-\mathrm{Sb}$ dumbbells have been implicated in the low thermal conductivity of the $\mathrm{Zn}_{4} \mathrm{Sb}_{3}$ phase. ${ }^{46}$

The low temperature $\alpha$-phase and high temperature $\beta$-phase of $\mathrm{Zn}_{4} \mathrm{Sb}_{3}$ were considered in this work. The unit cell of $\beta-\mathrm{Zn}_{4} \mathrm{Sb}_{3}$ is rhombohedral (space group $R \overline{3} C$ ) and contains three distinct atomic positions ( $36 \mathrm{Zn}, 18 \mathrm{Sb} 1$, and $12 \mathrm{Sb} 2$ amounting to a $\mathrm{Zn}_{3.6} \mathrm{Sb}_{3}$ stoichiometry). The atoms on the $\mathrm{Sb} 1$ site are free $\mathrm{Sb}^{3-}$ while those on the $\mathrm{Sb} 2$ site form $\left(\mathrm{Sb}_{2}\right)^{4-}$ dimers, according to the Zintl-Klemm concept. ${ }^{47}$ The problem (a)

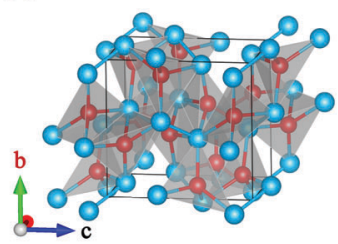

(c)

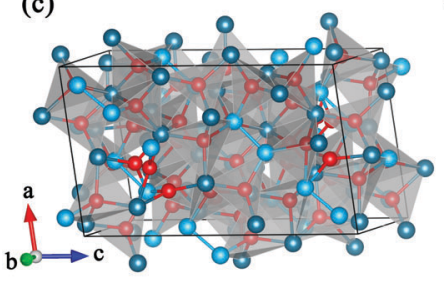

(e)

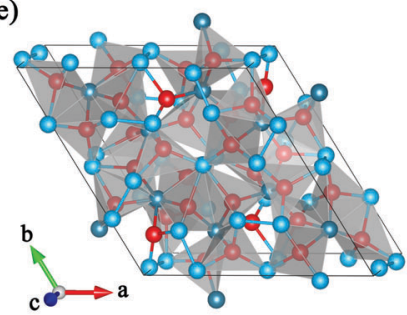

(b)

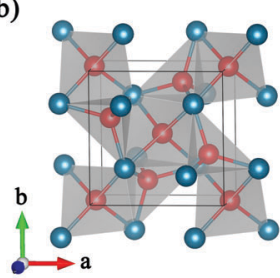

(d)

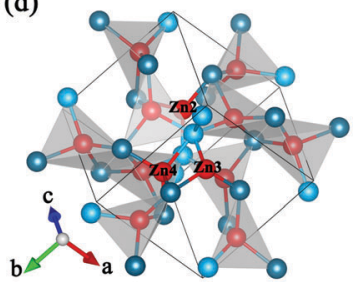

(f)

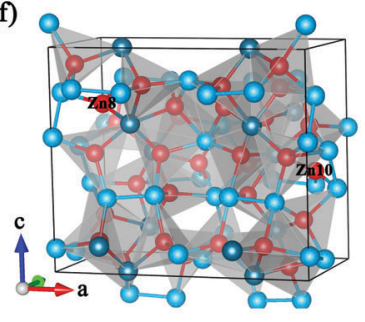

\section{- $\mathbf{Z n} \odot \mathbf{S b}^{2-} \odot \mathbf{S b}^{3-}$}

Fig. 2 Crystal structure of (a) $\mathrm{ZnSb}$, (b) predicted $\mathrm{Zn}_{3} \mathrm{Sb}_{2}$ structure with CALYPSO, (c) $\alpha-\mathrm{Zn}_{4} \mathrm{Sb}_{3}$, (d) $\beta-\mathrm{Zn}_{4} \mathrm{Sb}_{3}, \mathrm{Zn} 2, \mathrm{Zn} 3$, and $\mathrm{Zn} 4$ are interstitial atoms, (e) $\alpha-\mathrm{Zn}_{8} \mathrm{Sb}_{7}$, (f) $\beta-\mathrm{Zn}_{8} \mathrm{Sb}_{7}, \mathrm{Zn} 8, \mathrm{Zn} 10$ are interstitial atoms. posed by apparent deviation from the nominal composition was solved by Snyder et $a l^{23}$ They found a high degree of disorder in the $\mathrm{Zn}$ substructure of $\beta-\mathrm{Zn}_{4} \mathrm{Sb}_{3}$, and in particular interstitial $\mathrm{Zn}$ atoms distributed on three partially occupied general sites in space group $R \overline{3} C$ (36f). Additionally, the regular framework position (also 36f) displayed a considerable occupational deficiency with only $0.89-0.9$ of the sites filled. Based on this, the framework of $\beta-\mathrm{Zn}_{4} \mathrm{Sb}_{3}$ for our calculations was constructed in the following way: ${ }^{48,49}$ first, a rhombohedral primitive cell was built with full occupancy of $\mathrm{Zn} 1, \mathrm{Sb} 1$ and $\mathrm{Sb} 2$ sites resulting in the $\mathrm{Zn}_{12} \mathrm{Sb}_{10}$ stoichiometry. Then we removed two $\mathrm{Zn}$ atoms from the main site (Zn1) and inserted three different $\mathrm{Zn}$ interstitials in the $\mathrm{Zn} 2$, Zn3 and $\mathrm{Zn} 4$ sites, respectively, while maximizing the distance between interstitial atoms to minimize the total energy. ${ }^{25}$ We considered all the possible configurations and chose the lowest total energy one. The final structure is shown in Fig. 2 d. Below about $250 \mathrm{~K} \beta-\mathrm{Zn}_{4} \mathrm{Sb}_{3}$ transforms into $\alpha-\mathrm{Zn}_{4} \mathrm{Sb}_{3}$, which somewhat surprisingly has a completely ordered structure with a crystallographic composition of $\mathrm{Zn}_{13} \mathrm{Sb}_{10}$ for fully occupied sites. ${ }^{19}$ The structure of $\alpha-\mathrm{Zn}_{4} \mathrm{Sb}_{3}$ is triclinic, but is often described with a $C$-centred cell (i.e. $C \overline{1})$. Here, the interstitial $\mathrm{Zn}$ atoms are located on two fully occupied sites, and the main $\mathrm{Zn}$ site is also fully occupied (Fig. 2c). ${ }^{18}$

$\beta-\mathrm{Zn}_{8} \mathrm{Sb}_{7}$ is a new metastable compound whose synthesis was reported in $2015 .{ }^{20}$ It crystallizes in a noncentrosymmetric orthorhombic space group Pmn21. Twenty-four $\mathrm{Zn}$ atoms are distributed over 6 fully occupied crystallographic sites, while the remaining $8 \mathrm{Zn}$ atoms are distributed over 4 sites with partial occupancies. It contains $10\left(\mathrm{Sb}_{2}\right)^{4-}$ dumbbells and 8 isolated $\mathrm{Sb}^{3-}$ anions, which is similar to $\mathrm{Zn}_{13} \mathrm{Sb}_{10}$. Each $\mathrm{Zn}$ atom is surrounded by distorted tetrahedra with sharing of common edges. The distances of the reported $\mathrm{Zn} 7-\mathrm{Zn} 8$ and $\mathrm{Zn} 9-\mathrm{Zn} 10$ sites are in the range of 1.2-1.4 $\AA$. This indicates that only one of two possible $\mathrm{Zn}$ atoms is present. Thus, we use one $\mathrm{Zn} 8$ atom to replace the nearest $\mathrm{Zn} 7$ atom, similarly for $\mathrm{Zn} 9$ and $\mathrm{Zn10}$. The final crystal structure with the lowest total energy is shown in Fig. 2f. The $\alpha-\mathrm{Zn}_{8} \mathrm{Sb}_{7}$ phase was reported as $\mathrm{Zn}_{1+\delta} \mathrm{Sb}$ (with $\delta=0.068$ ) with a space group $P \overline{1} .^{21}$ It has an ordered structure with an ideal stoichiometry of $\mathrm{Zn}_{8} \mathrm{Sb}_{7}$ and few $\mathrm{Zn}$ defects in it. We adopt its ideal full occupancy structure in our calculation (Fig. 2e).

We performed variable-cell prediction simulations using the PSO methodology for $\mathrm{Zn}_{3} \mathrm{Sb}_{2}$ at $0 \mathrm{GPa}$. The lowest energy structure was chosen, as shown in Fig. 2b. Its crystal structure is tetragonal (space group $P \overline{4} 21 m, Z=2$ ), with lattice parameters $a=7.090 \AA$ and $c=4.777 \AA$. Sb occupies the $4 \mathrm{e}$ $(1.134,0.634,0.738)$ sites, while $\mathrm{Zn}$ atoms are at the $4 \mathrm{e}$ sites $(1.134,0.634,0.738)$ and $2 \mathrm{~d}$ sites $(0,0,0.5)$. All $\mathrm{Sb}$ in this structure are isolated $\mathrm{Sb}^{3-}$ anions, with no bonds between $\mathrm{Sb}$ atoms. We note that this structure differs from the hexagonal and cubic structures of $\mathrm{Mg}_{3} \mathrm{Sb}_{2}$ and $\mathrm{Ca}_{3} \mathrm{Sb}_{2}$. Each $\mathrm{Sb}$ atom is exclusively surrounded by six $\mathrm{Zn}$ atoms (at distances between 2.65 and $2.82 \AA$, similar to the known structures for $\beta-\mathrm{Zn}_{4} \mathrm{Sb}_{3} 2.7-2.8 \AA$, for $\alpha-\mathrm{Zn}_{4} \mathrm{Sb}_{3} 2.67-2.75 \AA$, for $\beta-\mathrm{Zn}_{8} \mathrm{Sb}_{7} 2.64-$ $2.87 \AA$, and $2.56-2.92 \AA$ for the $\alpha-\mathrm{Zn}_{8} \mathrm{Sb}_{7}$ phase). Each $\mathrm{ZnSb}_{4}$ 
tetrahedron shares a common $\mathrm{Sb}$ atom with another five tetrahedra.

It is perhaps surprising that $\mathrm{Zn}_{3} \mathrm{Sb}_{2}$ has a different structure than hexagonal $\mathrm{Mg}_{3} \mathrm{Sb}_{2}$. However, we note that the electronegativity of $\mathrm{Zn}$ is significantly higher than $\mathrm{Mg}$, which suggests chemical differences. We carefully checked the result by performing all-electron calculations for $\mathrm{Zn}_{3} \mathrm{Sb}_{2}$ in the fully relaxed $\mathrm{Mg}_{3} \mathrm{Sb}_{2}$ structure and our predicted tetragonal ground state structure. These calculations were done using the general potential linearized augmented planewave (LAPW) $\operatorname{method}^{50}$ as implemented in the WIEN2k code. ${ }^{51}$ We used highly converged basis sets based on the accurate LAPW augmentation, plus local orbitals to treat semicore states. We added additional local orbitals to improve the accuracy for the $\mathrm{Zn}$ d state. ${ }^{52}$ Spinorbit was included in these calculations. We find that our predicted tetragonal structure is lower in energy by $0.25 \mathrm{eV}$ per formula unit. This means that the tetragonal structure is strongly preferred over the $\mathrm{Mg}_{3} \mathrm{Sb}_{2}$ structure for $\mathrm{Zn}_{3} \mathrm{Sb}_{2}$.

We calculated the formation energy with respect to the elements for six configurations. As plotted in Fig. 3, the formation enthalpies of all the configurations are negative. In order to analyze phase stability at $0 \mathrm{~K}$, we constructed the convex hull of formation enthalpy, as illustrated by the solid lines in Fig. 3. Here we included the SOC effect. $\mathrm{ZnSb}$ is the most stable system with a formation energy of approximately $27 \mathrm{meV}$ per atom. The data shown for the interstitial $\beta$-phase structures are for the lowest formation energy structure out of all those that we built. A structure whose formation enthalpy lies above the convex hull, which can be considered as a global stability line, is metastable or unstable. The formation enthalpies of $\mathrm{Zn}_{4} \mathrm{Sb}_{3}, \mathrm{Zn}_{8} \mathrm{Sb}_{7}$ and $\mathrm{Zn}_{3} \mathrm{Sb}_{2}$ are above the convex hull. As expected, the interstitial frameworks of $\beta$-phases are less stable than those of the ordered $\alpha$-phases, but they still have a negative energy of formation ( $4.5 \mathrm{meV}$ per atom for $\beta-\mathrm{Zn}_{4} \mathrm{Sb}_{3}, 6 \mathrm{meV}$ per atom for $\left.\beta-\mathrm{Zn}_{8} \mathrm{Sb}_{7}\right)$. Surprisingly the $\alpha$-phase is unstable with respect to the decomposition into $\mathrm{ZnSb}$ and $\mathrm{Zn} .{ }^{21,53}$ The predicted structure of $\mathrm{Zn}_{3} \mathrm{Sb}_{2}$ has a little lower formation enthalpy

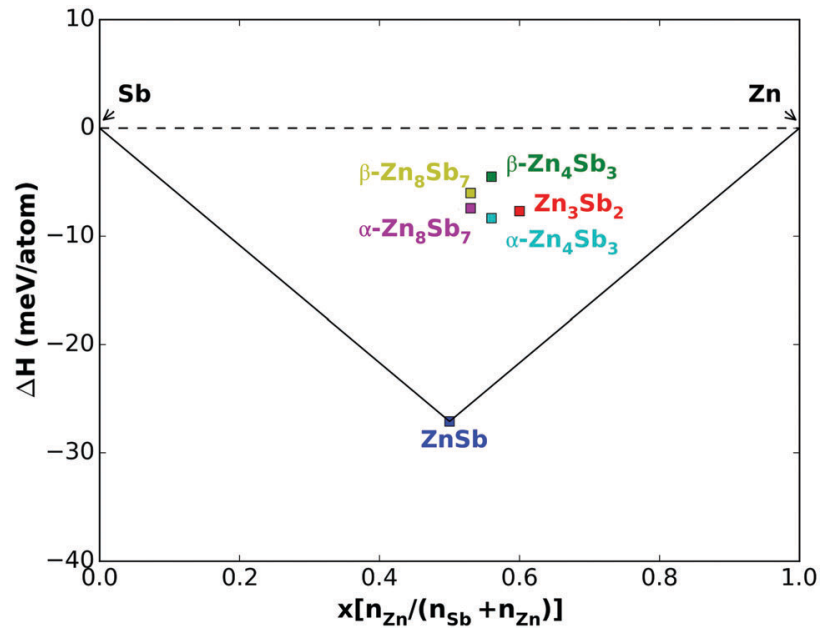

Fig. 3 Calculated enthalpy of formation as a function of $\mathrm{Zn}$ concentration.
( $8 \mathrm{meV}$ per atom). Without the SOC the formation energies are a little lower. But this does not change the stability situation for the $\mathrm{Zn}-\mathrm{Sb}$ binary system.

\section{Electronic and transport properties}

The calculated band structures and total and projected density of states (DOS) obtained with MBJ potential are shown in Fig. 4-9a. The Fermi levels are set to the top of the valence band. The results show that the MBJ method performs consistently better than PBE-GGA and produces band gaps that are in excellent agreement with the experimental values. This is in accord with prior results for other non-d-electron semiconductors. ${ }^{54,55}$ All the band structures shown are indirect band gap except $\beta-\mathrm{Zn}_{8} \mathrm{Sb}_{7}$. The PDOS show that the valence bands are formed from $\mathrm{Zn} \mathrm{p}, \mathrm{Sb}$ s, and $\mathrm{Sb}$ p hybridized states. The conduction band is characterized mainly by 4 s-electrons of zinc which are hybridized with 5p-electrons of antimony. The total density of states show a similar shape and magnitude of the valence band edges of $\mathrm{Zn}_{4} \mathrm{Sb}_{3}$ and $\mathrm{Zn}_{8} \mathrm{Sb}_{7}$, which suggests a similar hole carrier effective mass and similar electronic properties. It is remarkable that in spite of the different $\mathrm{Zn}-\mathrm{Sb}$ ratios and crystal structures, all these compounds show similar electronic structures. This is an example of the robustness of the Zintl concept for the $\mathrm{Zn}$-Sb system, specifically the balance between the $\mathrm{Zn}: \mathrm{Sb}$ and the $\mathrm{Sb}^{3-}: \frac{1}{2}\left(\mathrm{Sb}_{2}\right)^{4-}$ ratios.

Here we focus on the p-type, since experimentally all these compounds form as the p-type. We performed calculations of the thermopower as a function of temperature and the p-type
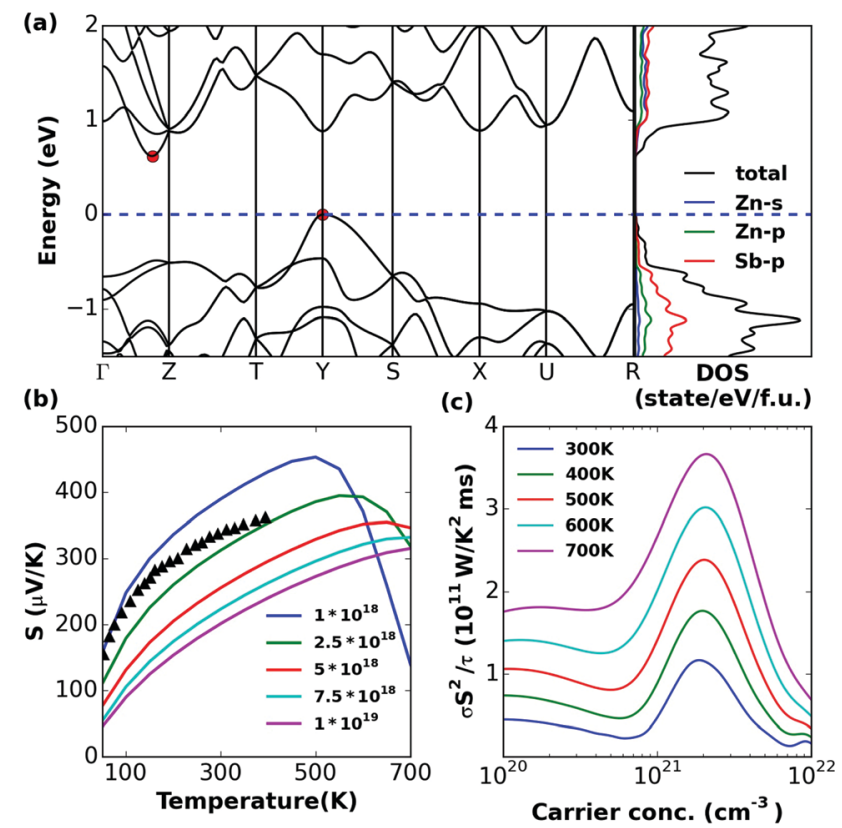

Fig. 4 Calculated (a) band structure and (partial) density of states, (b) thermopower $(S)$ versus temperature at different carrier concentrations (with different colorful lines), and (c) power factor divided by scattering time $\left(\sigma S^{2} / \tau\right)$ versus carrier concentration at different temperatures (with different colorful lines) for $\mathrm{ZnSb}$. In (a) the band edges are marked by red circles and in (b) experimental values (ref. 56) are shown with triangles for comparison. 
(a)

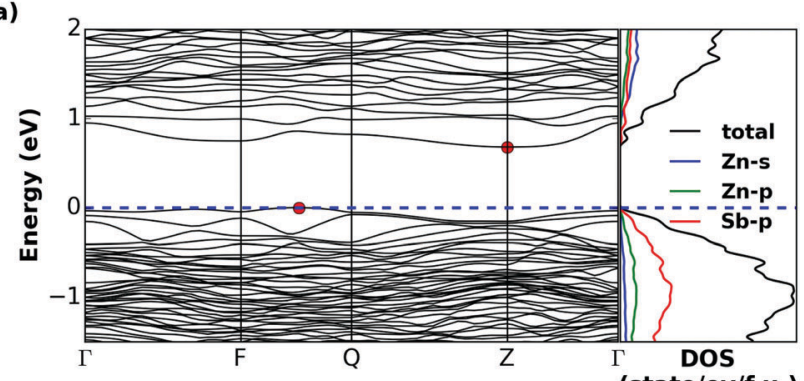

(b)

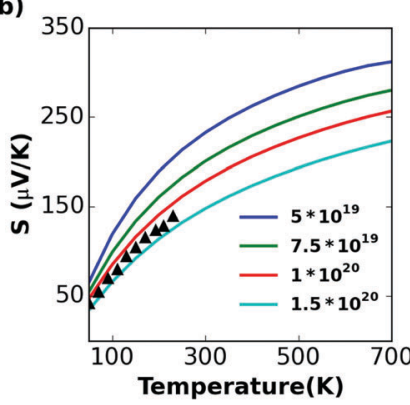

(c)

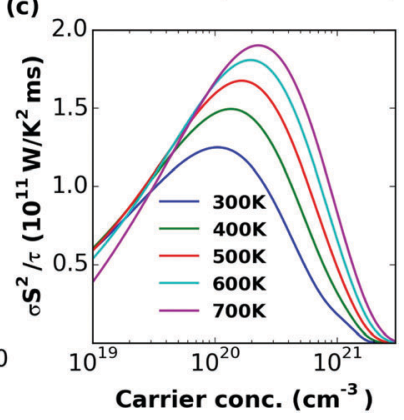

Fig. 5 Calculated (a) band structure and (partial) density of states, (b) $S$ and (c) $\sigma S^{2} / \tau$ of $\alpha-\mathrm{Zn}_{4} \mathrm{Sb}_{3}$. Experimental values reported in ref. 57 are shown with triangles for comparison.
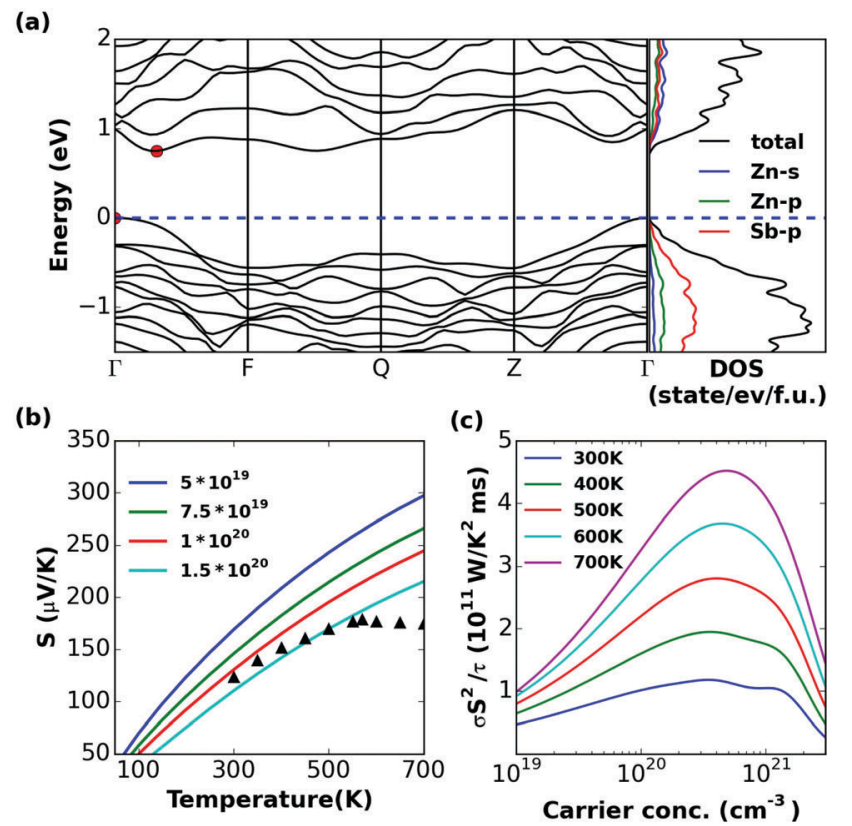

Fig. 6 Calculated (a) band structure and (partial) density of states, (b) $S$ (c) $\sigma S^{2} / \tau$ of $\beta-\mathrm{Zn}_{4} \mathrm{Sb}_{3}$. Experimental values reported in ref. 16 are plotted with triangles.

doping level with Boltzmann theory in the CSTA with rigid bands. We also show $S^{2} \sigma / \tau$ as a function of carrier concentration at different temperatures. This is the power factor divided by the scattering time, $\tau$, which is an undetermined parameter that can depend on both doping and temperature. Generally $\tau$ decreases with both temperature and carrier concentration, though the carrier concentration dependence

(a)

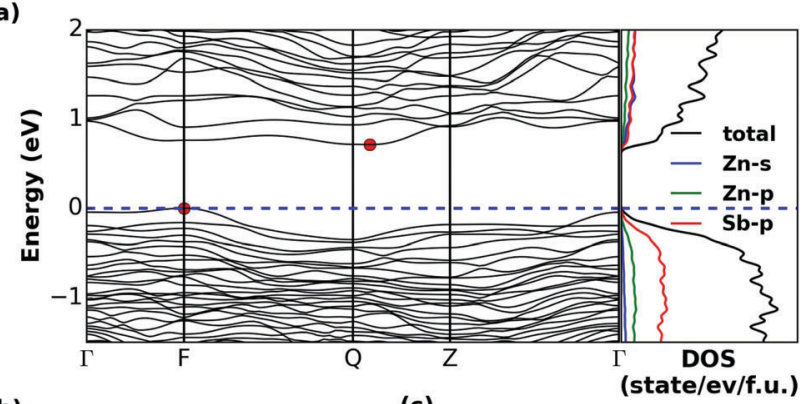

(b)

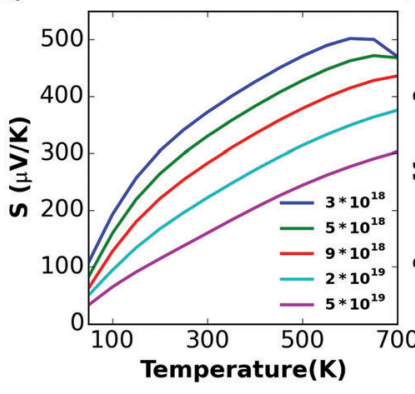

(c)

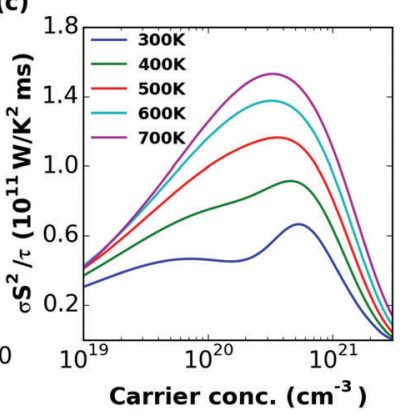

Fig. 7 Calculated (a) band structure and (partial) density of states, (b) $S$ and (c) $\sigma S^{2} / \tau$ of $\alpha-\mathrm{Zn}_{8} \mathrm{Sb}_{7}$.
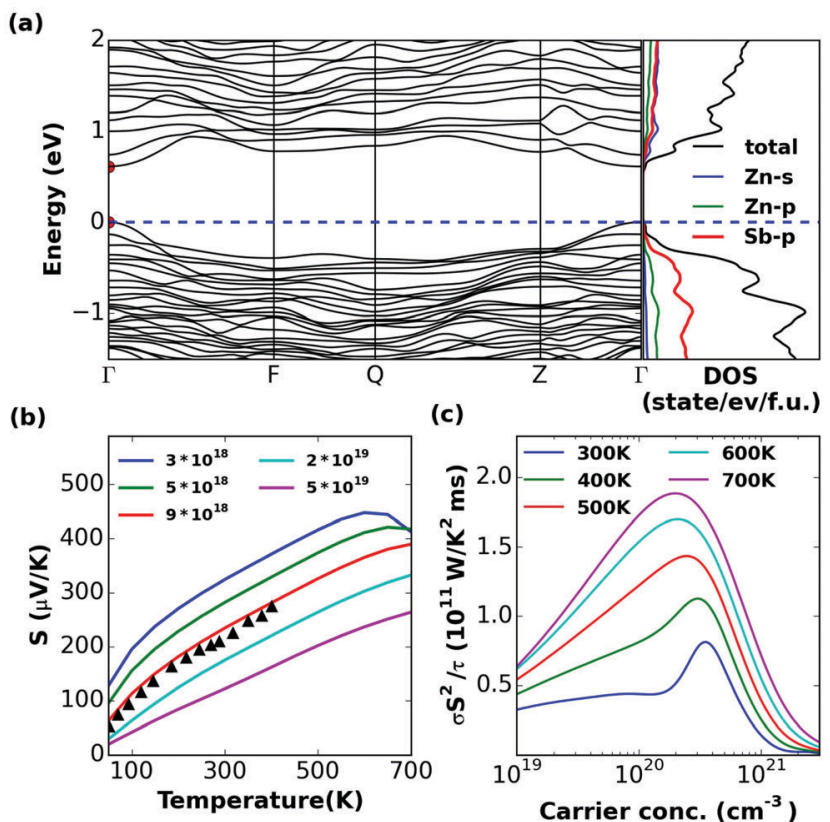

(c)

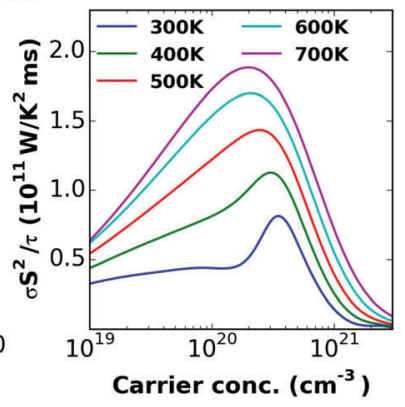

Fig. 8 Calculated (a) band structure and (partial) density of state, (b) $S$ (c) $\sigma S^{2} / \tau$ of $\beta-\mathrm{Zn}_{8} \mathrm{Sb}_{7}$. Experimental values reported in ref. 20 are shown with triangles for comparison.

is often weaker than the $\tau$ dependence. The validity of these approaches can be established by studying the agreement between theoretical results and experimental data shown in Fig. 4-6 and 8 and is discussed further in the text.

\section{ZnSb}

The valence bands are nearly parabolic at the band edge. We show the thermopower of $\mathrm{ZnSb}$ at carrier concentrations 
(a)

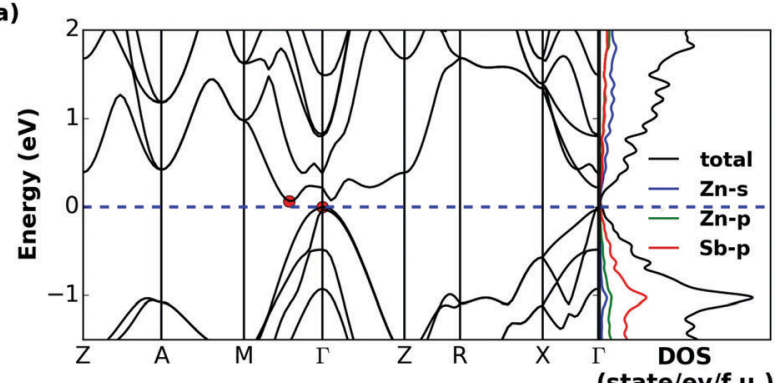

(b)

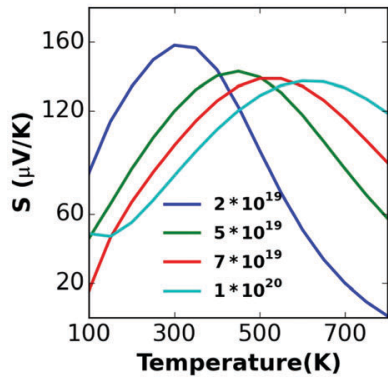

(c)

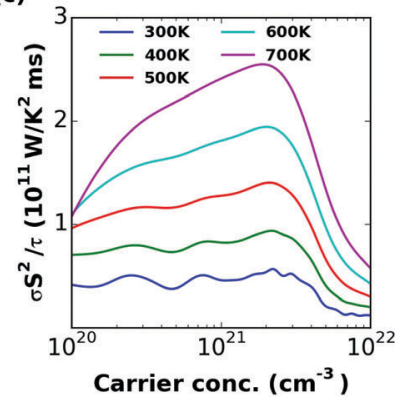

Fig. 9 Calculated (a) band structure and (partial) density of states, (b) $S$ and (c) $\sigma S^{2} / \tau$ of $\mathrm{Zn}_{3} \mathrm{Sb}_{2}$.

$\left(10^{18}-10^{19} \mathrm{~cm}^{-3}\right)$, which are in general accord with experimental data ${ }^{56}$ although the experimental carrier concentration needs to be established in order to determine how close the accord is (Fig. 4b). It should be noted that in $\mathrm{ZnSb}$ it has been proven that it is difficult to achieve hole concentrations above $10^{19} \mathrm{~cm}^{-3}$. The peak $\sigma S^{2} / \tau$ lies in the range of $1 \times 10^{20}$ to $4 \times 10^{20} \mathrm{~cm}^{-3}$ (Fig. 4c). It is highly likely that $Z T$ for $\mathrm{ZnSb}$ can be significantly increased if the carrier concentration can be increased.

\section{$\mathrm{Zn}_{4} \mathrm{Sb}_{3}$}

At the edge of the valence bands, we find heavier bands for the $\alpha$ phase than the $\beta$ phase, resulting in higher DOS near the VBM (Fig. 5 and 6a), which should lead to a little larger thermopower in p-type materials. They display similar band gap $E_{\mathrm{g}}=0.68$ and $0.77 \mathrm{eV}$, respectively. The latter agrees well with the experimental value $E_{\mathrm{g}}=0.8 \mathrm{eV}^{39}$ for the $\beta$ phase. The thermopower value of the $\alpha$ phase is indeed a little higher than the $\beta$ phase in our calculation (Fig. 5 and 6b). Experimental measurements of $S$ for $\alpha-\mathrm{Zn}_{4} \mathrm{Sb}_{3}$ are only available for temperatures below $250 \mathrm{~K} .{ }^{57}$ One can see that our interstitial structures can get thermopowers in accord with the experiment again with the caveat of the carrier concentration. ${ }^{16}$ The doping level at 300-700 K corresponding to the peak $\sigma S^{2} / \tau$ lies in the range of $1 \times 10^{20}$ to $4 \times 10^{20} \mathrm{~cm}^{-3}$ for the $\alpha$ phase and $2 \times 10^{20}$ to $9 \times 10^{20} \mathrm{~cm}^{-3}$ for the $\beta$ phase (Fig. 5 and $6 \mathrm{c}$ ), again implying that the peak $Z T$ is likely to be at high carrier concentration.

$\mathrm{Zn}_{8} \mathrm{Sb}_{7}$

The band structure of $\alpha-\mathrm{Zn}_{8} \mathrm{Sb}_{7}$ shows that it is an indirect band gap semiconductor, with $E_{\mathrm{g}}=0.71 \mathrm{eV}$. Disordered $\beta-\mathrm{Zn}_{8} \mathrm{Sb}_{7}$ has a direct band gap, with $E_{\mathrm{g}}=0.61 \mathrm{eV}$. They have very similar transport properties. The doping level at 300-700 K corresponding to the peak $\sigma S^{2} / \tau$ lies in the range of $1 \times 10^{20}$ to $4 \times 10^{20} \mathrm{~cm}^{-3}$ for both $\alpha$ and $\beta$ phases (Fig. 7 and 8c). It was found that the thermopower values of the disordered $\beta$-phase and the ordered $\alpha$-phase of $\mathrm{Zn}_{4} \mathrm{Sb}_{3}$ and $\mathrm{Zn}_{8} \mathrm{Sb}_{7}$ were very similar. Hence, the key to the outstanding thermoelectric properties of the $\beta$-phase lies in the exceptionally low thermal conductivity, which is as low as that for glass-like materials combined with its favorable electronic properties.

\section{$\mathrm{Zn}_{3} \mathrm{Sb}_{2}$}

A narrow band gap of $0.06 \mathrm{eV}$ separates the valence states to the conduction states. The electronic structure of $\mathrm{Zn}_{3} \mathrm{Sb}_{2}$ exhibits the charge carrier pockets with light bands near the VBM and CBM, which leads to low thermopower. As predicted the light band at the edge of bands indeed leads to a low thermopower for $\mathrm{Zn}_{3} \mathrm{Sb}_{2}$ (Fig. 9b). The very small gap suggests that this phase will not be a good thermoelectric at least at room temperature and above. This is also seen in the thermopower, which shows strong bipolar effects.

\section{Conclusions}

The $\beta$-phases of $\mathrm{Zn}_{8} \mathrm{Sb}_{7}$ and $\mathrm{Zn}_{4} \mathrm{Sb}_{3}$ have complex crystal structures. These structures challenge crystallography because of the presence of site disorder for the $\mathrm{Zn}$ atoms. In this work, we have proposed structural models of $\beta$-phases, which are close to experimental studies. All binary $\mathrm{Zn} / \mathrm{Sb}$ compounds are narrow-gap semiconductors. We have analysed the phase stability, electronic structure, optical property of $\mathrm{ZnSb}$ and transport properties by means of first principles density functional theory and Boltzmann transport calculations. Additionally, we have searched for the $\mathrm{Zn}_{3} \mathrm{Sb}_{2}$ structure using the PSO method. This yields a structure different from the hexagonal structure of $\mathrm{Mg}_{3} \mathrm{Sb}_{2}$, reflecting the different chemistries of $\mathrm{Zn}$ and $\mathrm{Mg}$. In terms of the formation energy, this phase is comparable to $\mathrm{Zn}_{8} \mathrm{Sb}_{7}$ and $\mathrm{Zn}_{4} \mathrm{Sb}_{3}$. In thermodynamic stability analysis, they are all metastable phases.

A similar shape and magnitude of the total density of states in the valence band edge of $\mathrm{Zn}_{4} \mathrm{Sb}_{3}$ and $\mathrm{Zn}_{8} \mathrm{Sb}_{7}$ lead to similar thermopower values. The thermopower data and transport properties from our calculations show that $\mathrm{Zn}_{3} \mathrm{Sb}_{2}$ should not be a good candidate for thermoelectric materials at room temperature and above. The existing experimental results show the best performance for $\beta-\mathrm{Zn}_{4} \mathrm{Sb}_{3}$. This material has a reasonable band gap, shows multiple valence bands sufficiently near the band edge to influence the thermoelectric behavior and importantly has a low thermal conductivity characteristic of the $\beta$-phases. However, our results also show that there is still room for improvement in $Z T$ of the $\beta$ phases by optimization of carrier concentration. A comparison of the compounds in terms of the balance between $\sigma / \tau$ and $S$ at $400 \mathrm{~K}$ and a carrier concentration of $10^{19} \mathrm{~cm}^{-3}$ as well as the doping dependence of $S$ is given in Fig. 10. 
(a)

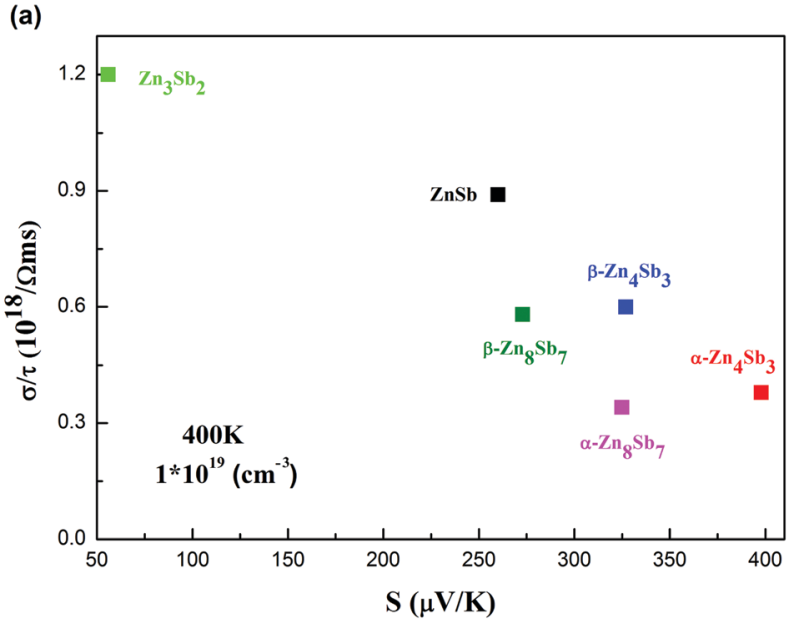

(b)

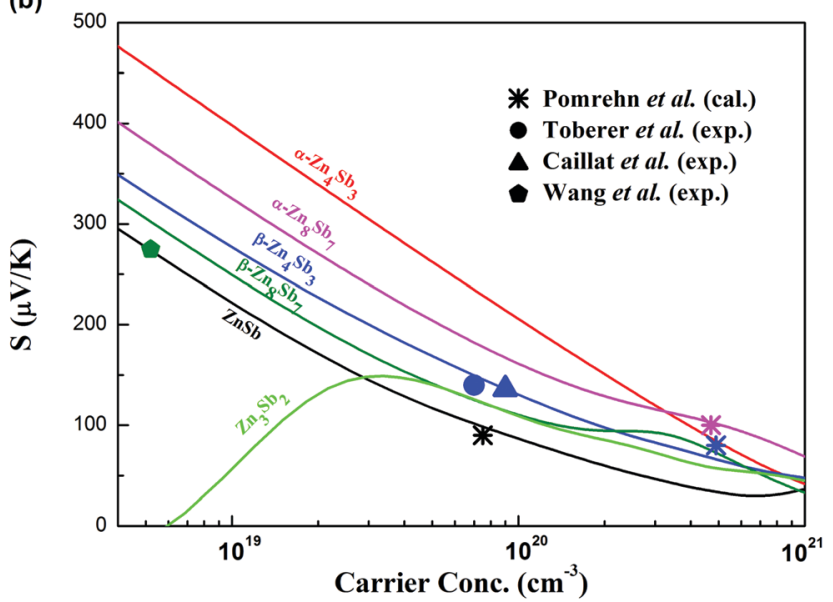

Fig. 10 (a) Electrical conductivity divided by scattering time $(\sigma / \tau)$ versus thermopower (S) calculated at a temperature of $400 \mathrm{~K}$ and a carrier concentration of $10^{19} \mathrm{~cm}^{-3}$, and (b) thermopower versus carrier concentration calculated at a temperature of $400 \mathrm{~K}$ for different phases of the $\mathrm{Zn}-\mathrm{Sb}$ materials. In (b) the available experimental and calculated data from the previous work by Pomrehn et al. (ref. 29), Toberer et al. (ref. 57), Caillat et al. (ref. 16), and Wang et al. (ref. 20) are shown for comparison; for the scatters, consistent colors with those of lines are used for the same phases.

Finally it is interesting to note that although some gaps are small, all the compounds are semiconducting. This is a reflection of the Zintl concept in those phases, especially the balance between the $\mathrm{Zn}: \mathrm{Sb}$ and $\mathrm{Sb}^{3-}: \frac{1}{2}\left(\mathrm{Sb}_{2}\right)^{4-}$ ratios reflecting stabilization or destabilization of $\left(\mathrm{Sb}_{2}\right)^{4-}$ polyanions according to the amount of charge available from the $\mathrm{Zn}^{2+}$ cations. We note that several of these phases are underdoped for thermoelectric performance. The flexibility of these compounds in terms of carrier compensation via the $\mathrm{Sb}^{3-}: \frac{1}{2}\left(\mathrm{Sb}_{2}\right)^{4-}$ ratio poses a challenge for achieving the optimum doping level. The solution to this problem may lead to significantly better $Z T$ in the $\mathrm{Zn}-\mathrm{Sb}$ phases.

\section{Acknowledgements}

The authors acknowledge funding support from the Recruitment Program of Global Youth Experts in China and special funds for talent development in Jilin Province. Part of calculations was performed in the high performance computing center of Jilin University. Work of D. J. S is supported by S3TEC an Energy Frontier Research Center funded by the Department of Energy, Office of Science, Basic Energy Sciences under award DE-SC0001299/DE-FG02-09ER46577.

\section{References}

1 H. J. Goldsmid and R. W. Douglas, Br. J. Appl. Phys., 1954, 5, 386-390.

2 C. Wood, Rep. Prog. Phys., 1988, 51, 459-540.

3 L. E. Bell, Science, 2008, 321, 1457-1461.

4 G. Chen, M. S. Dresselhaus, G. Dresselhaus, J.-P. Fleurial and T. Caillat, Int. Mater. Rev., 2003, 48, 45-66.

5 J. Yang, L. Xi, L. Wu, X. Shi, L. Chen, J. Yang, W. Zhang, C. Uher and D. J. Singh, NPJ Comput. Mater., 2016, 2, 15015.

6 L. Muchler, F. Casper, B. Yan, S. Chadov and C. Felser, Phys. Status Solidi RRL, 2012, 7, 91-100.

7 H. Shi, D. Parker, M. H. Du and D. J. Singh, Phys. Rev. Appl., 2015, 3, 014004.

8 Y. Xu, Z. Gan and S. C. Zhang, Phys. Rev. Lett., 2014, 112, 226801.

9 G. Xing, J. Sun, K. P. Ong, X. Fan, W. Zheng and D. J. Singh, APL Mater., 2016, 4, 053201.

10 M. Sasaki, H. Negishi and M. Inoui, J. Appl. Phys., 1986, 59, 796-802.

11 S. Walia, R. Weber, S. Sriram, M. Bhaskaran, K. Latham, S. Zhuiykov and K. Kalantar-Zadeh, Energy Environ. Sci., 2011, 4, 3558-3564.

12 S. Walia, R. Weber, S. Balendhran, D. Yao, J. T. Abrahamson, S. Zhuiykov, M. Bhaskaran, S. Sriram, M. S. Strano and K. Kalantar-Zadeh, Chem. Commun., 2012, 48, 7462-7464.

13 S. Walia, S. Balendhran, P. Yi, D. Yao, S. Zhuiykov, M. Pannirselvam, R. Weber, M. S. Strano, M. Bhaskaran, S. Sriram and K. Kalantar-Zadeh, J. Phys. Chem. C, 2013, 117, 9137-9142.

14 S. Walia, R. Weber, K. Latham, P. Petersen, J. T. Abrahamson, M. S. Strano and K. Kalantar-Zadeh, Adv. Funct. Mater., 2011, 21, 2072-2079.

15 S. Walia, S. Balenhran, H. Nili, S. Zhuiykov, G. Rosengarten, Q. H. Wang, M. Bhaskaran, S. Sriram, M. S. Strano and K. Kalantar-Zadeh, Prog. Mater. Sci., 2013, 50, 1443-1489.

16 T. Caillat, J.-P. Fleurial and A. Borshchevsky, J. Phys. Chem. Solids, 1997, 58, 1119-1125.

17 K. E. Almin, Acta Chem. Scand., 1948, 2, 400-407.

18 Y. Mozharivskyj, Y. Janssen, J. L. Harringa, A. Kracher, A. O. Tsokol and G. J. Miller, Chem. Mater., 2006, 18, 822-831.

19 J. Nylén, M. Andersson, S. Lidin and U. Häussermann, J. Am. Chem. Soc., 2004, 126, 16306-16307.

20 J. Wang and K. Kovnir, J. Am. Chem. Soc., 2015, 137, 12474-12477.

21 C. S. Birkel, E. Mugnaioli, T. Gorelik, U. Kolb, M. Panthöfer and W. Tremel, J. Am. Chem. Soc., 2010, 132, 9881-9889. 
22 F. Adjadj, E.-d. Belbacha, M. Bouharkat and A. Kerboub, J. Alloys Compd., 2006, 419, 267-270.

23 G. J. Snyder, M. Christensen, E. Nishibori, T. Caillat and B. B. Iversen, Nat. Mater., 2004, 3, 458-463.

24 P. H. M. Böttger, S. Diplas, E. Flage-Larsen, Ø. Prytz and T. G. Finstad, J. Phys.: Condens. Matter, 2011, 23, 265502.

25 F. Cargnoni, E. Nishibori, P. Rabiller, L. Bertini, G. J. Snyder, M. Christensen, C. Gatti and B. B. Iversen, Chem. - Eur. J., 2004, 10, 3861-3870.

26 S.-G. Kim, I. I. Mazin and D. J. Singh, Phys. Rev. B: Condens. Matter Mater. Phys., 1998, 57, 6199-6203.

27 S. Wang, H. Li, D. Qi, W. Xie and X. Tang, Acta Mater., 2011, 59, 4805-4817.

28 U. Häussermann and A. S. Mikhaylushkin, Dalton Trans., 2010, 39, 1036-1045.

29 G. S. Pomrehn, E. S. Toberer, G. J. Snyder and A. van de Walle, J. Am. Chem. Soc., 2011, 133, 11255-11261.

30 A. N. Qiu, L. T. Zhang and J. S. Wu, Phys. Rev. B: Condens. Matter Mater. Phys., 2010, 81, 035203.

31 K. Niedziolka, R. Pothin, F. Rouessac, R. M. Ayral and P. Jund, J. Phys.: Condens. Matter, 2014, 26, 365401.

32 P. H. M. Bottger, S. Diplas, E. Flage-Larsen, O. Prytz and T. G. Finstad, J. Phys.: Condens. Matter, 2011, 23, 265502.

33 P. E. Blöchl, Phys. Rev. B: Condens. Matter Mater. Phys., 1994, 50, 17953-17979.

34 G. Kresse and J. Furthmüller, Phys. Rev. B: Condens. Matter Mater. Phys., 1996, 54, 11169-11186.

35 J. P. Perdew and A. Zunger, Phys. Rev. B: Condens. Matter Mater. Phys., 1981, 23, 5048-5079.

36 L. Sham and M. Schlüter, Phys. Rev. Lett., 1983, 51, 1888-1891.

37 F. Tran and P. Blaha, Phys. Rev. Lett., 2009, 102, 226401.

38 H. Komiya, K. Masumoto and H. Fan, Phys. Rev., 1964, 133, A1679-A1684.

39 M. Vaida, N. Duteanu and I. Grozescu, J. Serb. Chem. Soc., 2016, 81, 323-332.

40 G. K. Madsen and D. J. Singh, Comput. Phys. Commun., 2006, 175, 67-71.
41 B. DeWitt and H. Seltz, J. Am. Chem. Soc., 1939, 61, 3170-3173.

42 M. Boström and S. Lidin, J. Alloys Compd., 2004, 376, 49-57.

43 Y. Wang, J. Lv, L. Zhu and Y. Ma, Phys. Rev. B: Condens. Matter Mater. Phys., 2010, 82, 094116.

44 Y. Wang, J. Lv, L. Zhu and Y. Ma, Comput. Phys. Commun., 2012, 183, 2063-2070.

45 A. Jain, S. P. Ong, G. Hautier, W. Chen, W. D. Richards, S. Dacek, S. Cholia, D. Gunter, D. Skinner, G. Ceder and K. A. Persson, APL Mater., 2013, 1, 011002.

46 W. Schweika, R. P. Hermann, M. Prager, J. Person and V. Keppens, Phys. Rev. Lett., 2007, 99, 125501.

47 G. A. Papoian and R. Hoffmann, Angew. Chem., Int. Ed., 2000, 39, 2408-2448.

48 E. Toberer, K. Sasaki, C. Chisholm, S. Haile, W. Goddard and G. Snyder, Phys. Status Solidi RRL, 2007, 1, 253-255.

49 S. Wang, X. Tan, G. Tan, X. She, W. Liu, H. Li, H. Liu and X. Tang, J. Mater. Chem., 2012, 22, 13977-13985.

50 D. J. Singh and L. Nordstrom, Planewaves Pseudopotentials and the LAPW Method, Springer, Berlin, 2nd edn, 2006.

51 P. Blaha, K. Schwarz, G. Madsen, D. Kvasnicka and J. Luitz, in WIEN2k, An Augmented Plane Wave+Local Orbitals Program for Calculating Crystal Properties, K. Schwarz, Tech. Univ. Wien, Austria, 2001.

52 D. Singh, Phys. Rev. B: Condens. Matter Mater. Phys., 1991, 43, 6388-6392.

53 A. S. Mikhaylushkin, J. Nylén and U. Häussermann, Chem. - Eur. J., 2005, 11, 4912-4920.

54 D. J. Singh, Phys. Rev. B: Condens. Matter Mater. Phys., 2010, 82, 205102.

55 D. Koller, F. Tran and P. Blaha, Phys. Rev. B: Condens. Matter Mater. Phys., 2011, 83, 195134.

56 D. Eklöf, A. Fischer, Y. Wu, E.-W. Scheidt, W. Scherer and U. Häussermann, J. Mater. Chem. A, 2013, 1, 1407-1414.

57 T. Souma, G. Nakamoto and M. Kurisu, J. Alloys Compd., 2002, 340, 275-280. 